\title{
Molecular spectroscopy with a terahertz quantum-cascade laser by illumination-induced frequency tuning
}

\author{
Tasmim Alam, ${ }^{1}$ Martin Wienold, ${ }^{1,2}$, Heinz-Wilhelm Hübers ${ }^{1,2}$ \\ ${ }^{1}$ German Aerospace Center (DLR), Institute of Optical Sensor Systems, Rutherfordstr. 2, 12489 Berlin, Germany \\ ${ }^{2}$ Humboldt-Universität zu Berlin, Dept. of Physics, Newtonstr. 15, 12489 Berlin, Germany
}

\begin{abstract}
We report on molecular spectroscopy with a terahertz quantum-cascade laser (QCL). The frequency of the QCL is tuned by illuminating one of its facets using a near-infrared laser. A tuning range of $17 \mathrm{GHz}$ is achieved for continuous-wave operation.
\end{abstract}

\section{INTRODUCTION}

Terahertz (THz) spectroscopy plays an important role in astronomy and planetary research since many molecular absorption and emission lines of interest fall into this spectral range. For high-resolution heterodyne or laser absorption spectroscopy, it is essential to tune the frequency of the source over a specific frequency range. For $\mathrm{THz}$ quantum-cascade lasers (QCLs), which are employed as spectroscopic sources above $3 \mathrm{THz}$, tuning is typically realized either by ramping the driving current or varying the heat sink temperature. The drawbacks of these techniques are the limited tuning range and the rather slow speed for temperature tuning. In order to overcome these limitations, several techniques have been demonstrated during the last few years. Among them are the use of external optical cavities [1], mechanical tuning of cavities [2], and electrical frequency tuning [3]. A promising technique for the frequency tuning of THz QCLs is the illumination of the rear facet with a near-infrared diode laser [4], which has been introduced recently. Here, we report on molecular spectroscopy with a THz QCL, by tuning its frequency via facet illumination.

\section{EXPERIMENTAL SETUP AND RESULTS}

Figure 1 depicts the schematic setup for the measurement. The employed QCL emits a single mode at $3.3 \mathrm{THz}$ with up to $3 \mathrm{~mW}$ of output power when operated in continuous-wave (cw) mode in a He-flow cryostat at $37 \mathrm{~K}$. The QCL is based on a single-plasmon waveguide and the active region is described in Ref. 5. In a first step, the current and temperature tuning coefficients were determined. For this calibration, a current ramp was applied, and the transmission signal through a gas cell was determined.

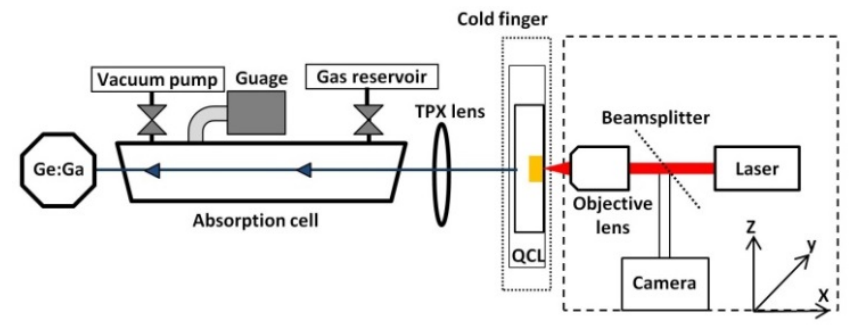

Fig. 1: Schematic setup for the facet illumination of a QCL with a diode laser
Due to its rich absorption spectrum, methanol was used as molecular species. Temperature and current tuning coefficients of $-220 \mathrm{MHz} / \mathrm{K}$ and $+19 \mathrm{MHz} / \mathrm{mA}$, respectively, were determined. A near-infrared diode laser was used to illuminate one of the facets of the QCL. For illumination, we employed a home-made confocal microscope setup with a camera. The laser spot was focused to the facet with an infinity corrected objective lens with a magnification factor of 10 and a working distance of $33.5 \mathrm{~mm}$. For alignment, an $\mathrm{XYZ}$ translation stage was used. At the beginning, we carried out tests with a single mode diode laser. The laser emits at 807 $\mathrm{nm}$ with a lasing threshold current of $107 \mathrm{~mA}$. It is coupled to a polarization-maintaining fiber (PM780-HP) with a $4.5 \mu \mathrm{m}$ core diameter. A constant current of $360 \mathrm{~mA}$ was applied to the QCL, and the diode laser was tuned from below threshold up to the maximum operating current (387 mA). The emission from the other facet of the QCL was collimated by a TPX lens and measured with a Ge:Ga photo-conductive detector after passing through a $60-\mathrm{cm}$-long gas cell. Absorption spectra are acquired within $80 \mathrm{~ms}$ time scale by measuring the detector signal as a function of the diode laser current. The tuning range achieved by this method in the current configuration is $5.65 \mathrm{GHz}$ for $100 \mathrm{~mW}$ of incident power. In order to increase the tuning range, the single mode diode laser was replaced by a high power diode laser. The laser emits at $809 \mathrm{~nm}$ with a lasing threshold current of $1.05 \mathrm{~A}$ and it is coupled to a 200$\mu \mathrm{m}$ core-diameter multi-mode fiber by means of an HP-SMA connector. By analyzing the obtained methanol absorption spectrum, we identified a tuning range of $17 \mathrm{GHz}$ for $1 \mathrm{~W}$ incident optical power. Figure 2 shows a respective absorption spectrum of methanol at a pressure of $1 \mathrm{hPA}$.

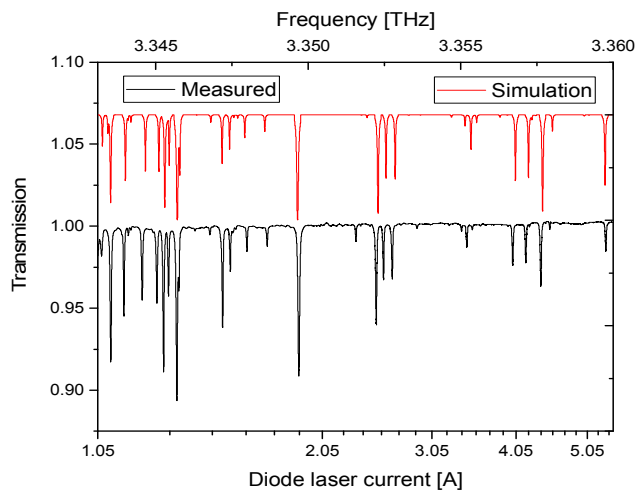

Fig. 2: Transmission signal (methanol, at a pressure of $1 \mathrm{hPa}$ ) as a function of frequency/current.

From the tuning behavior, we found that for temperature tuning the absorption lines are shifting toward lower frequencies, while for diode laser tuning the lines are shifting toward higher frequencies. From this observation, it is clear 
that the observed blue shift is not driven by heating, but rather due to the generation of electron hole plasma which changes the refractive index as well as the absorption coefficient close to the facet [4]. The data plotted in Fig. 3 depicts quantitatively the frequency shift as a function of the incident optical power. The frequency shift $\Delta v_{s}$ follows the relation

$$
\Delta v_{s}=A \sqrt{P}
$$

where $P$ denotes the incident optical power and $A$ is a scaling factor. The square root dependence on the incident power is consistent with bimolecular recombination of electron-hole pairs as the dominant recombination process for the induced free-carrier plasma.

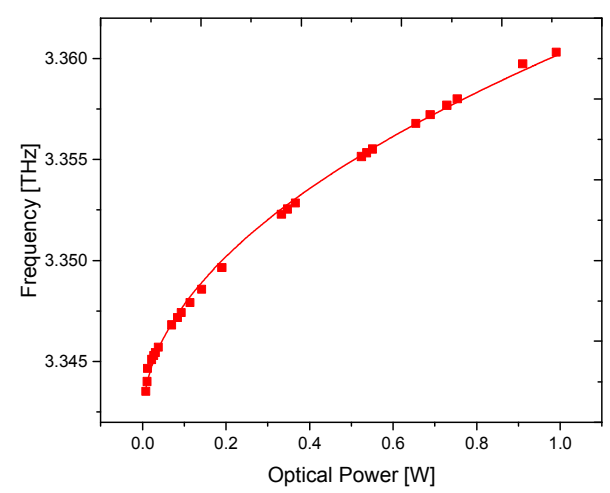

Fig. 3. Frequency shift as a function of optical power. The solid line refers to Eq. 1.

To emphasize the importance of our approach for practical applications, the QCL was also operated inside a mechanical cryocooler. Due to the cooler vibrations, it was difficult to focus the laser spot with a $10 x$ objective. With a $2 x$ objective in combination with a single mode diode laser, we achieved a tuning range of $3.35 \mathrm{GHz}$ for $100 \mathrm{~mW}$ injected optical power. A detailed study of this method and its further improvement is currently under investigation.

\section{SUMMARY}

We have demonstrated molecular spectroscopy with a $3.3 \mathrm{THz}$ QCL by tuning the frequency via facet illumination with a diode laser. A tuning range of $17 \mathrm{GHz}$ was obtained with $1 \mathrm{~W}$ of injected optical power, which enabled the acquisition of highly resolved molecular spectra on a millisecond time scale.

\section{ACKNOWLEDGEMENTS}

We thank L. Schrottke and H. T. Grahn (Paul-Drude-Institut, Berlin) for providing the lasers and for helpful discussions. Part of this work was supported Deutsche Forschungsgemeinschaft (DFG) (HU848/5-1). T. Alam acknowledges the support by the Helmholtz Research School on Security Technologies.

\section{References}

[1]. J. Xu, J. M. Hensley, D. B. Fenner, R. P. Green, L. Mahler, A. Tredicucci, M. G. Allen, F. Beltram, H. E. Beere, and D. A. Ritchie, "Tunable terahertz quantum cascade lasers with an external cavity", Appl. Phys. Lett., vol. 91, pp. 121104, 2007.

[2]. Q. Qin, B. S. Williams, S. Kumar, J. L. Reno, and Q. Hu, "Tuning a terahertz wire laser" Nature Photon, vol. 3, pp. 732, 2009.

[3]. K. Ohtani, M. Beck, and J. Faist, "Electrical laser frequency tuning by three terminal terahertz quantum cascade lasers", Appl. Phys. Lett., vol. 104, pp. $011107,2014$.

[4]. M. Hempel, B. Röben, L. Schrottke, H.-W. Hübers, and H. T. Grahn, "Fast continuous tuning of terahertz quantum-cascade lasers by rear-facet illumination", Appl. Phys. Lett., vol. 108, pp. 191106, 2016.

[5]. L. Schrottke, M. Wienold, R. Sharma, X. Lü, K. Biermann, R. Hey, A. Tahraoui, H. Richter, H-W. Hübers, and H. T. Grahn, "Quantum-cascade lasers as local oscillators for heterodyne spectrometers in the spectral range around 4.745 THz", Semiconductor Science and Technology., vol. 28, pp. 035011, 2013. 